\title{
Ultrafast structural changes measured by time-resolved X-ray diffraction
}

\author{
J. Larsson ${ }^{1}$, P.A. Heimann ${ }^{2}$, A.M. Lindenberg ${ }^{1}$, P.J. Schuck ${ }^{1}$, P.H. Bucksbaum ${ }^{3}$, R.W. Lee ${ }^{4}$, H.A. Padmore ${ }^{2}$, J.S. Wark ${ }^{5}$, \\ R.W. Falcone ${ }^{1,6, *}$ \\ ${ }^{1}$ Physics Department, University of California at Berkeley, Berkeley, CA 94720, USA \\ (E-mail: jorgen@physics.berkeley.edu) \\ ${ }^{2}$ Advanced Light Source, Accelerator and Fusion Research Division, Lawrence Berkeley National Laboratory, Berkeley, CA 94720, USA \\ ${ }^{3}$ Physics Department and Center for Ultrafast Optical Science, University of Michigan, Ann Arbor, MI 48109, USA \\ ${ }^{4}$ Lawrence Livermore National Laboratory, Livermore, CA 94551, USA \\ ${ }^{5}$ Physics Department, Clarendon Laboratory, University of Oxford, Oxford OX13PU, UK \\ ${ }^{6}$ Center for Beam Physics, Accelerator and Fusion Research Division, Lawrence Berkeley National Laboratory, Berkeley, CA 94720, USA
}

Received: 17 April 1998/Accepted: 17 April 1998

\begin{abstract}
High-intensity X-ray pulses from third-generation synchrotron sources make it possible to study the temporal dynamics of rapidly evolving materials. We report a study of rapid and reversible disordering of the structure of an InSb crystal induced by an ultrashort laser pulse. A novel crosscorrelation detection technique is described, which allowed us to observe rapid changes in X-ray diffraction that occur on a time-scale of less than 2 ps.
\end{abstract}

Time-resolved X-ray diffraction experiments were undertaken at the Advanced Light Source synchrotron. We irradiated an InSb crystal with a 100 fs laser pulse, and probed the irradiated region with a $70 \mathrm{ps} \mathrm{X}$-ray pulse. A rapid reduction in the X-ray diffraction signal was observed, followed by recovery on a timescale of about $100 \mathrm{~ns}$. In order to distinguish between various mechanisms that could cause the reduction in X-ray signal, ultrafast time resolution is required. We therefore developed a cross-correlation technique using two laser-irradiated crystals. This technique will allow studies with a temporal resolution limited only by the laser pulse duration. In our current work, we were limited by a low signal-to-noise ratio, which results in a temporal resolution of about 2 ps. Additionally, and from measurements of the angular dependence of the diffraction efficiency (rocking curve), we conclude that the ultrashort laser pulse induced reversible disorder, or some other phase transition, in the bulk InSb crystal.

\footnotetext{
${ }^{*}$ Corresponding author.
}

\section{Previous studies of crystalline structure dynamics}

The structural properties of materials following irradiation with ultrashort light pulses has been studied for two decades [1-11]. One motivation for this work has been the study of ultrafast disordering (e.g., melting) and other potential light-induced phase transitions (e.g., to novel ordered structures). Pump-probe techniques have generally been employed. On ultrafast timescales $(<1 \mathrm{ps})$, studies have involved time-resolved reflectivity and second-harmonic generation, both using optical laser pulses. Subpicosecond timescale is fundamental, and consistent with atomic motion on the scale of a typical molecular bond length. On longer timescales, electron [12,13] and X-ray [14-20] diffraction have provided insight into phenomena including thermallyinduced melting, shock propagation, strain, heat diffusion, crystal regrowth, and annealing.

As an example of ultrafast structural changes in materials, theoretical studies suggest that optical excitation of about $10 \%$ of the valence electrons in crystals with a diamond or zincblende structure should result in a structural instability which gives rise to ultrafast disordering on a time-scale shorter than the electron-phonon relaxation time [21-24]. It has also been speculated that such structural instabilities could result in a transient, ordered phase [24].

\section{Studies of InSb}

In our experiment, we examined the disordering of laserirradiated InSb. The material was electronic grade, crystalline wafer material with (111) orientation. InSb was chosen for several reasons. The first is that it is a well studied material. Phase transitions in InSb under pressure in a diamond anvil cell have been studied with X-ray diffraction [25-27]. 
A permanent phase transition under long-pulse laser irradiation has been observed [28]. Ultrafast carrier dynamics have been studied using probes in the optical and $\mathrm{THz}$ spectral regions [7, 29]. Carrier relaxation through Auger recombination has also been investigated [30]. A second consideration is that $\mathrm{InSb}$ is a high-Z material with a short $\mathrm{X}$-ray absorption depth. Since the mechanism suggested for laser-induced phase transitions requires a relatively high electron density, the pumplaser penetration depth could be severely shortened by freecarrier absorption, independent of the optical properties of the material prior to excitation. Consequently, phase-transitions would occur in a relatively thin layer near the surface. Since $\mathrm{X}$-rays typically penetrate deeply into materials, the X-ray probe could then pass through a laser-disordered layer and Bragg-scatter significantly from unperturbed material. However, in InSb, photoabsorption rapidly attenuates the X-ray intensity in from the surface. (An alternate way of matching the laser and X-ray penetration depths is to use thin layers of material grown epitaxially on a special substrate.)

Additionally, we estimate that in InSb and other smallbandgap materials, interband absorption should exceed freecarrier absorption for the laser fluences used in our experiment. Absorption in $\mathrm{InSb}$ at the laser wavelength of $800 \mathrm{~nm}$ corresponds to a $1 / \mathrm{e}$ penetration depth of $100 \mathrm{~nm}$ [31]. The $\mathrm{X}$-ray photoabsorption depth at $5 \mathrm{KeV}$ is $2300 \mathrm{~nm}$, but in the Bragg-scattering geometry at an angle of $19^{\circ}$ we estimate the absorption depth to be $370 \mathrm{~nm}$, and therefore on-scale with the depth of laser-excited material. In InSb the sound speed

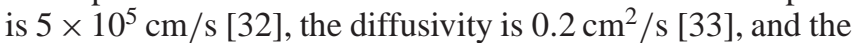
thermal expansion coefficient is $5 \times 10^{-6} \mathrm{~K}^{-1}$ [33].

\section{Setup for pump/probe experiments}

Our experiments utilize synchrotron radiation from a bending magnet beamline at the Advanced Light Source (ALS) which is described in [34]. This X-ray source has a broad spectrum of radiation extending to about $12 \mathrm{KeV}$. The divergence of the beam is set to $0.4 \mathrm{mrad}$ by an aperture. X-rays are monochromatized and sagittally focused to a line using a bent Si wafer cut in the (111) plane. The wafer is bent into a cylinder by two rectangular springs; a crystal length-to-width ratio of about $4: 1$ limits anticlastic bending, and the width of the line focus is typically $100 \mu \mathrm{m}$. The diffraction plane is vertical in order to have the polarization of the X-rays perpendicular to the plane of incidence, and the focusing in the horizontal plane yields monochromatic X-rays. The Bragg angle is $22^{\circ}$, which gives a photon energy of $5 \mathrm{KeV}$ for $\mathrm{Si}(111)$. A horizontal $1 \mathrm{~mm}$ slit is placed between the bent Si crystal and the InSb sample in order to define a probed region within the laser illuminated area. The angle between the laser and X-rays is made small $(0.1 \mathrm{rad})$ in order to reduce the geometric time delay between pump and probe beams to less than $200 \mathrm{fs}$.

A Ti: $\mathrm{Al}_{2} \mathrm{O}_{3}$ laser system produces pulses with a wavelength of $800 \mathrm{~nm}$ and a duration of $100 \mathrm{fs}$ at a repetition rate of $1 \mathrm{KHz}$; it is synchronized to the electron storage ring with jitter of less than $10 \mathrm{ps}$. The delay between the laser and the $\mathrm{X}$-ray pulse is varied using an optical-delay line in the laser beam path. For the cross-correlation experiments, a beamsplitter is introduced and a second delay line is used to vary the relative timing of laser pulses incident on the two InSb crystals. The laser beams are focused on the InSb samples at a fluence of about $30 \mathrm{~mJ} / \mathrm{cm}^{2}$. Samples are mounted on goniometric cradles which have a resolution and repeatability of $5 \mu \mathrm{rad}$. Diffracted X-rays are detected by an avalanche photodiode (APD) with a response time of $10 \mathrm{~ns}$. Signals are recorded using a digital oscilloscope or a gated-integrator connected to a computer.

Using a calibrated X-ray photodiode operated in the linear regime, we measure about $10 \mathrm{X}$-ray photons per pulse in a diffraction spot from the InSb sample. In order to obtain a sufficiently good signal-to-noise ratio, data must be acquired over multiple pulses. We thus rely on recovery of the sample between laser pulses (in less than $1 \mathrm{~ms}$ ). It is therefore important to determine if any laser induced phasetransition is followed by regrowth and, if so, to determine the structure of the re-grown crystal. Regrowth of semiconductor and metal crystals following laser illumination has been well studied $[35,36]$. At the fluence used in this study we do not observe any irreversible damage of the InSb or any significant drop in the diffracted X-ray signal, even after typical experimental runs totaling more than $15 \mathrm{~min}$. However, at $20 \%$ higher fluence, we observe irreversible damage within less than a second. This damage is visible to the eye and results in a lowering of the time-averaged X-ray diffraction efficiency by about $30 \%$. The laser energy on target was measured using a power meter, and the spot size on target was determined using a CCD camera; we estimate the uncertainty in the absolute fluence to be less than $30 \%$.

Temporal structure of the ALS X-ray pulses in our experiments results from an isolated, high-current electron bunch in the storage ring, which is separated by about $16 \mathrm{~ns}$ from a pulse-train of about 250 low-current pulses which are each separated by 2 ns. The 16 ns time-separation allowed us to select the intense X-ray pulse from the isolated electron bunch using the APD detector and gated-integrator.

\section{Time-resolved X-ray diffraction studies}

We first made use of the pulse-train in order to study (relatively) slower timescale phenomena, by recording the signal from the APD using a digitizing oscilloscope. Figure 1 shows

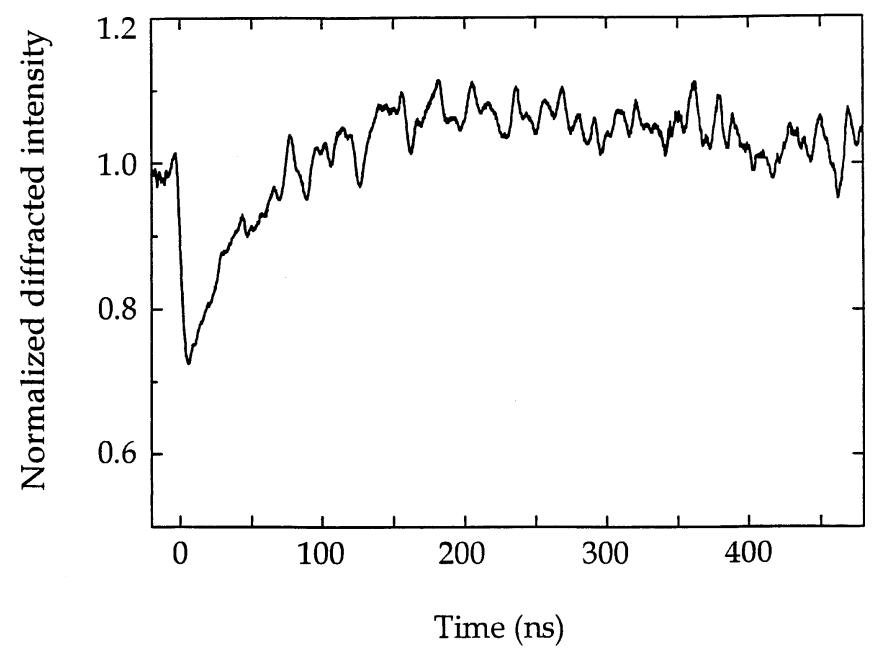

Fig. 1. Diffracted X-ray intensity from pulsed-laser irradiated $\mathrm{InSb}$ as a function of time 
the resulting diffracted X-ray intensity as a function of time, with $10 \mathrm{~ns}$ resolution. We observe a rapid, 30\% decrease in diffracted intensity and a recovery on a timescale of $100 \mathrm{~ns}$, followed by a slight increase relative to the signal before laser irradiation. We attribute this increase to the crystal initially being set slightly off the Bragg peak. At the maximum in the signal intensity, the peak of the rocking curve has temporarily shifted into resonance with the selected X-ray wavelength, due to lattice expansion at an elevated temperature reached following regrowth and during diffusive cooling.

In order to investigate the observed decrease in Braggscattering efficiency with higher temporal resolution than shown in Fig. 1, we employed a scanned, pump-probe technique. In Fig. 2 the diffracted X-ray intensity is shown as a function of delay between the X-ray probe pulse and the scanned laser pulse. The drop in diffraction intensity measured here appears limited in time-resolution by the ALS-pulse duration, which is on the order of $80 \mathrm{ps}$ for the ALS operating conditions [37]. By differentiating this data, we see that the ALS pulse shape is recovered, as shown by the dashed trace in Fig. 2. From this measurement we conclude that the $\mathrm{X}$-ray diffraction efficiency drops with a characteristic time at least a factor 2 smaller than the ALS pulse duration, i.e., $<40$ ps. The data in Fig. 2 comprises about $10^{5}$ laser pulses at the repetition rate of $1 \mathrm{KHz}$.

The rapid drop and recovery in Bragg-scattered intensity could be related to several possible effects, including disorder (melting) and regrowth, lattice expansion and relaxation, ablation of the top layer, and phase transitions to different structures. As described below, we interpret the data as follows: (1) as the laser interacts with the crystal, the top layer disorders; (2) underlying layers are heated, causing strain; (3) as heat diffuses away from the surface layer, the crystal structure regrows at an elevated temperature with expanded lattice spacing; (4) as the heated region equilibrates with the bulk of the crystal through thermal diffusion, the original lattice order and spacing is restored.

In order to eliminate lattice expansion and other acoustictimescale effects as explanations of the initial decrease in Bragg-scattering, we need to observe the effect with higher

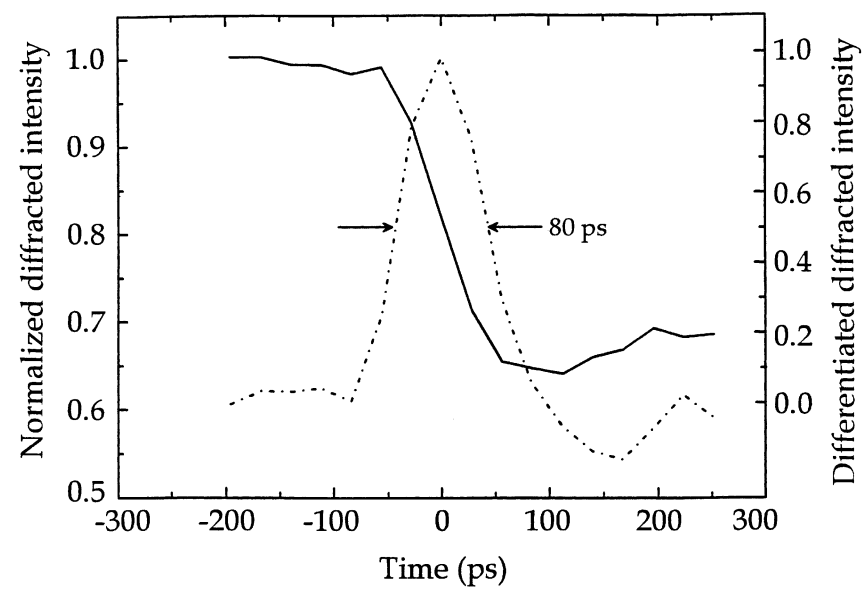

Fig. 2. Diffracted X-ray intensity from pulsed-laser irradiated InSb, recorded as a function of delay between laser irradiation and the X-ray probe (solid line). The derivative of this data (dotted line) yields the expected shape for the ALS pulse. We conclude that the decrease in diffraction efficiency from the laser-irradiated crystal occurs in less than $40 \mathrm{ps}$ time resolution. The response of lattice expansion is limited by the sound speed; we can obtain a lower bound for the timescale of this effect by dividing the laser penetration depth $(100 \mathrm{~nm})$ by the sound speed $\left(5 \times 10^{5} \mathrm{~cm} / \mathrm{s}\right)$, yielding a response of $20 \mathrm{ps}$, which is faster than the resolution of the pump-probe technique described above. We therefore developed a cross-correlation technique which enabled measurements of changes in X-ray diffraction with temporal resolution that is fundamentally limited only by the laser pulse duration (100 fs). This cross-correlation technique is illustrated in Fig. 3. Here, we modify the shape of the original X-ray pulse from the ALS by diffracting it from a crystal that is irradiated by a short laser pulse. We subsequently probe that modified X-ray pulse by inducing a rapidly varying reflectivity in a second crystal which is irradiated by a second laser pulse. Data is obtained by scanning the second (near) step function over the first (near) step function. As shown by the solid line data in Fig. 4, we obtain a ramped signal (which levels off) proportional to the net diffracted energy through the system as a function of delay between the two laser pulses. The change of slope of this curve (its derivative is shown by the dotted line in Fig. 4) indicates a rapid timescale for the laser-induced reduction of Braggreflectivity in both crystals. The signal-to-noise ratio is currently limited by the photon flux at our beamline, and the data was smoothed with a filter corresponding to $(2 \mathrm{ps})^{-1}$ bandwidth; this currently limits the temporal resolution of the technique. However, we are able to rule out lattice expansion as the mechanism for the observed rapid reduction in Bragg-scattered intensity, since the data in Fig. 4 indicate

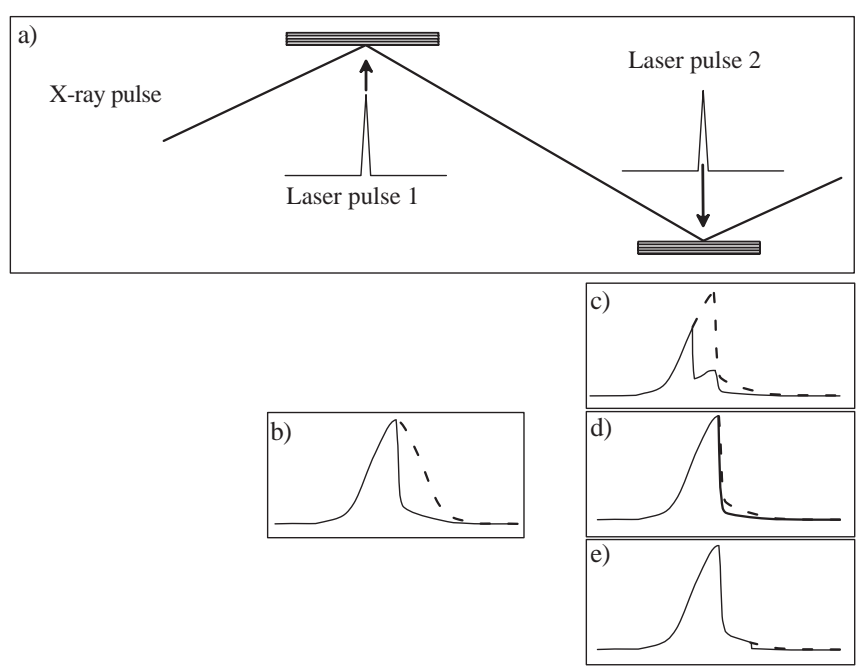

Fig. 3a-e. Cartoon explaining the cross-correlation technique used to obtain ultrafast time-resolution. a Geometry for the experiment. b The incident $\mathrm{X}$-ray pulse (dashed line) has a duration of $80 \mathrm{ps}$. The laser pulse irradiates the first crystal, introducing a rapid fall in the diffracted X-ray pulse shape. c-e A second laser pulse interacts with the second crystal, rapidly decreasing its diffraction efficiency. In c, the diffraction efficiency decrease occurs before the rapid fall in the incident X-ray pulse; in d, the diffraction efficiency decrease is simultaneous with the rapid fall in the incident $\mathrm{X}$-ray pulse; in e, the diffraction efficiency decrease occurs late in time. There is a large change in the diffracted energy through the system between c and $\mathbf{d}$, and a small change in the diffracted energy through the system between $\mathbf{d}$ and e. When the delay between the two laser pulses is scanned, a rapid change of slope in the integrated signal occurs when the crystals are simultaneously irradiated 


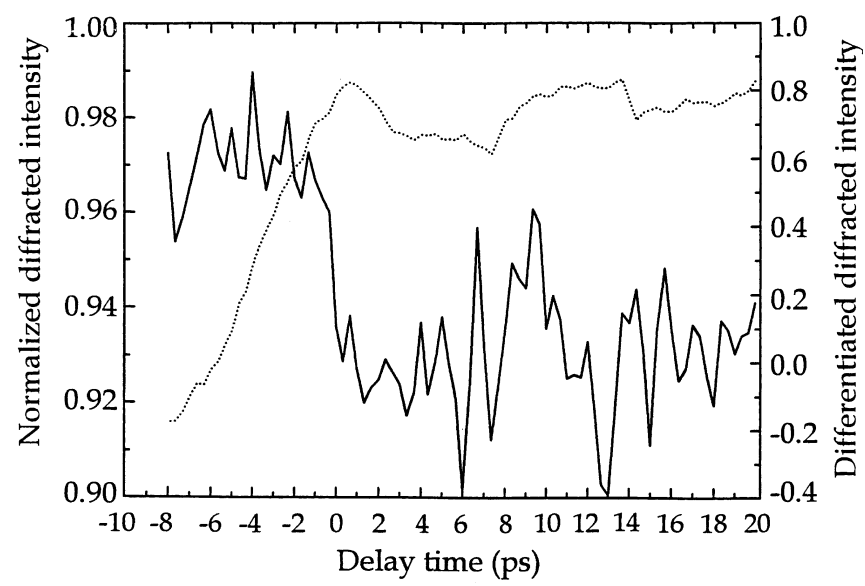

Fig. 4. Diffracted X-ray intensity as a function of the time delay between the laser pulse incident on the first and second crystals in the cross-correlation experiment (solid line). The timing of the laser pulse on the first crystal with respect to the ALS pulse is fixed, and the timing of the second laser pulse on the second crystal is varied. The sharpness of that change of slope (the derivative, shown as the dotted line) reveals the timescale for disordering, but temporal resolution is limited by a 2 ps smoothing filter

that the timescale of the reduction in the signal must be faster than 2 ps. We therefore invoke disordering (or other phase change) of the lattice as an explanation for the rapid reduction in the Bragg-scattered signal.

Another signature of lattice expansion would be a shift of the Bragg peak towards smaller angles. In Fig. 5 we show measured rocking curves for the InSb crystal, which indicate our sensitivity to such shifts. In particular, there is a shift due to the average heating of the wafer by the $30 \mathrm{~mW} / \mathrm{cm}^{2}$ laser intensity. This shift is determined by comparing the rocking curve measured with the laser turned off (solid line) with the rocking curve measured with the probe X-rays timed $250 \mathrm{ps}$ before the laser pulse, or equivalently, nearly $1 \mathrm{~ms}$

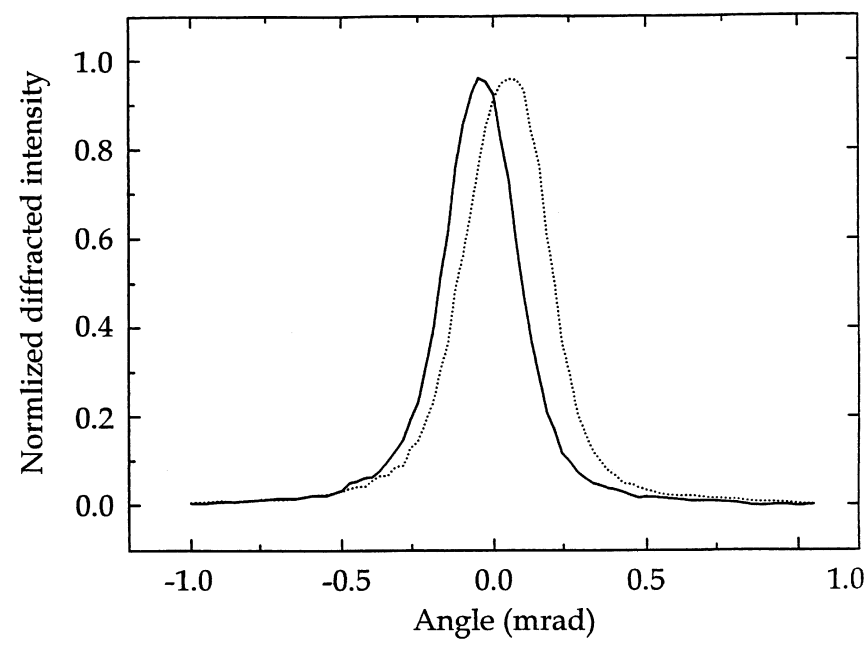

Fig. 5. Rocking curves for the (111) reflection of InSb. The solid line is the rocking curve when the laser is off. The dotted line is the rocking curve recorded when X-rays probe $250 \mathrm{ps}$ before the laser irradiates the crystal at its $1 \mathrm{KHz}$ repetition rate. Average heating by the laser is seen to result in a $50 \mathrm{~K}$ temperature rise of the sample after a laser pulse, given the $1 \mathrm{KHz}$ laser repetition rate (dotted line). A shift of approximately $80 \mathrm{mrad}$ is observed, corresponding to a temperature rise of the crystal of $50 \mathrm{~K}$ above room temperature due to average power heating. In Fig. 6 we compare the rocking curve taken with the probe $\mathrm{X}$-rays timed $250 \mathrm{ps}$ before the laser pulse (๑), equivalent to nearly a $1 \mathrm{~ms}$ after a laser pulse, with a rocking curve measured with the probe X-ray timed $250 \mathrm{ps}$ after the laser irradiates the sample $(\boldsymbol{\Delta})$. Here we measure negligible shift in the peaks. However, we do observe both an asymmetric pedestal on the low-angle side and a peak reduction of the rocking curve measured $250 \mathrm{ps}$ after the laser pulse, compared to the other curve. We interpret the asymmetric pedestal as diffraction from a strained layer below the disordered surface. Not shown is an additional rocking curve, measured with the $80 \mathrm{ps}$ X-ray probe coincident in time with the laser pulse, where we similarly see no angular shift in the position of the peak.

To obtain more detailed understanding of the shape and intensity of the measured rocking curves, we model the experiments by numerically solving the dynamical diffraction equations using the method detailed in [38]. In that model, a temperature distribution in the crystal is taken into account. Due to bulk material expansion, the lattice constant varies with depth in from the surface. We set the initial temperature distribution to be an exponential with a decay depth equal to the $1 / \mathrm{e}$ laser attenuation depth, where the top diffracting layers are at the melting temperature. Lattice expansion in the model is determined by the linear material expansion coefficient in the (111) direction. The dotted line in Fig. 6 shows the results of that model (convoluted with a Gaussian to obtain the experimental width for the crystal prior to laser irradiation). The best agreement for the shape of the experimental curve and the simulation was for a $1 / \mathrm{e}$ strain depth of $35 \mathrm{~nm}$. A disordered depth of $47 \mathrm{~nm}$ is deduced from the $12 \%$ drop in the integrated intensity. The integrated reflectivity of the strained crystal is negligibly different from the

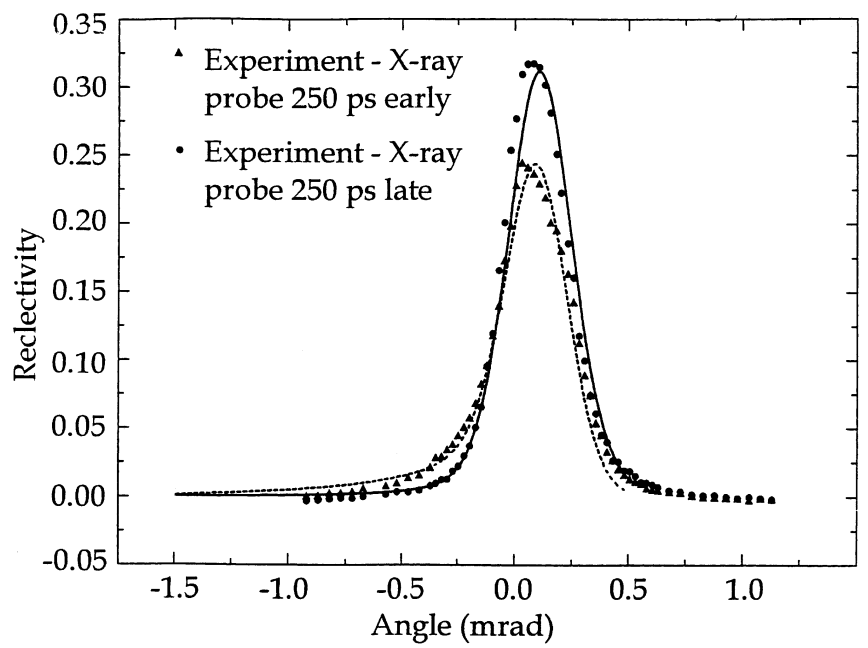

Fig. 6. Rocking curves for the (111) reflection of InSb, measured when the $\mathrm{X}$-ray pulse probes $250 \mathrm{ps}$ before the laser pulse $(\bullet)$, and when the X-ray pulse probes $250 \mathrm{ps}$ after the laser pulse $(\mathbf{\Delta})$. Model rocking curves, which have been normalized to the measured signal levels, are also shown, indicating the effects of disorder and strain in the crystal when X-rays probe $250 \mathrm{ps}$ after the laser pulse 
unstrained value; this can be understood by noting the relatively high X-ray absorption coefficients, which means that the kinematic and dynamical reflectivity are nearly identical. We conclude that the drop in integrated diffraction intensity is due to disordering of the structure near the surface.

\section{Conclusion}

We have observed a rapid phase transition in InSb by measuring time-resolved X-ray scattering from the laser-irradiated crystal. Disorder occurred on a timescale of $<2$ ps. The results are consistent with an study in which femtosecond optical pulses were used to probe laser-irradiated InSb [7]. In that work, optical properties of the material were observed to change on a timescale of $300 \mathrm{fs}$. We note that if these results are a manifestation of the same phase transition observed in our X-ray studies, it is expected that order-disorder transition times of much less than a picosecond could enable a novel gating scheme for switching ultrashort X-ray pulses out of longer duration pulses, such as those generated at the ALS. We have also shown that X-ray pulses with induced, ultrafast time structure are a suitable tool for the study of the temporal dynamics of condensed matter phase transitions. We note that we at present are not able to determine if the laser-induced, reversible disordering that occurs in InSb is thermally induced melting or a new type of optically-induced phase transition; improved time resolution will be required to investigate that issue. Employing the techniques described here in such work will benefit from the higher signal-to-noise available at more powerful synchrotron beamline devices, including wigglers and undulators, and the use of advanced X-ray optics.

Since this article was prepared, additional work relevant to the problems of ultrafast time-resolved X-ray scattering has appeared in: M. Wulff, F. Schottee, G. Naylor, D. Bourgeois, K. Moffat, G. Mopurou: Nucl. Instr. Meth. A 398, 69 (1997); C. Rischel, A. Rousse, I. Uschmann, P.-A. Albouy, J.-P. Geindre, P. Audebert, J.C. Gauthier, E. Förster, J.-L. Martin, A. Antonetti: Nature 390, 480 (1997); T. Gou, C. Rose-Petruck, R. Jimenez, F. Raksi, J. Squire, B. Walker, K.R. Wilson, C.P.J. Barty: "Picosecond-milliangstrom resolution dynamics by ultrafast x-ray diffraction" (SPIE Proceedings in press).

Acknowledgements. This work was supported by an Academic Research Instrumentation grant from the National Science Foundation, and by the Department of Energy through both Lawrence Livermore National Laboratory and Lawrence Berkeley National Laboratory. P.H.B. acknowledges support from the Miller Foundation of the University of California at Berkeley. J.L. acknowledges support from the Swedish Natural Science Research Council. J.L. is now with the Atomic Physics Division and the Division for Synchrotron Radiation Research at Lund University, P.O. Box 118, S-221 00 Lund, Sweden.

\section{References}

1. J.A. VanVechten, R. Tsu, F.W. Saris, D. Hoonhout: Phys. Lett. 74A, 417 (1979)

2. C.V. Shank, R. Yen, C. Hirlimann: Phys. Rev. Lett. 50, 454 (1983)

3. M.C. Downer, R.L. Fork, C.V. Shank: J. Opt. Soc. Am. B 2, 596 (1985)

4. H.K.W. Tom, G.D. Aumiller, C.H. Briton-Cruz: Phys. Rev. Lett. 60, 1438 (1988)

5. D.H. Reitze, X. Wang, H. Ahn, M.C. Downer: Phys. Rev. B 40, 11986 (1989)

6. K. Sokolowski-Tinten, J. Bialkowski, D. von der Linde: Phys. Rev. B 51, 14186 (1995)

7. J.L. Shumay, U. Höfer: Phys. Rev. B 53, 15878 (1996)

8. J. Solis, C.N. Afonso, S.C.W. Hyde, N.P. Barry, P.M.W. French: Phys. Rev. Lett. 76, 2519 (1996)

9. M.F. Becker, A.B. Buckman, R.M. Walser, T. Lepine, P. Georges, A. Brun: J. Appl. Phys. 29, 2404 (1996)

10. Y. Siegal, E.N. Gletzer, E. Mazur: Phys. Rev. B 49, 16403 (1994)

11. E.N. Glezer, Y. Siegal, L. Huang, E. Mazur: Phys. Rev. B 51, 9589 (1995)

12. G. Mourou, S. Williamson: Appl. Phys. Lett. 41, 44 (1982)

13. H.E. Elsayed-Ali, T.B. Norris, M.A. Pessot, G.A. Mourou: Phys. Rev. Lett 58, 1212 (1987)

14. R.R. Whitlock, J.S. Wark: Phys. Rev. B 52, 8 (1995)

15. J.S. Wark, N.C. Woolsey, R.R. Whitlock: Appl. Phys. Lett. 61, 651 (1992)

16. P. Chen, I.V. Tomov, P.M. Rentzepis: J. Chem. Phys. 104, 10001 (1996)

17. B.C. Larson, J.F. Barhorst: J. Appl. Phys. 51, 3181 (1980)

18. B.C. Larson, C.W. White, T.S. Noggle, J.F. Barhorst, D.M. Mills: Appl. Phys. Lett. 42, 282 (1982)

19. J.G. Lunney, P.J. Dobson, J.D. Harris, S.D. Tabatabaei, R.W. Eason: Opt. Commun. 58, 269 (1986)

20. J.R. Buschert, J.Z. Tischler, D.M. Mills, Q. Zhao, R. Corella: J. Appl. Phys. 66, 3523 (1989)

21. P. Stampfli, K.H. Bennemann: Phys. Rev. B 42, 7163 (1990)

22. P. Stampfli, K.H. Bennemann: Phys. Rev. B 46, 10686 (1992)

23. P. Stampfli, K.H. Bennemann: Phys. Rev. B 49, 7299 (1994)

24. P. Stampfli, K.H. Bennemann: Appl. Phys. A 60, 191 (1995)

25. M.D. Banus, M.C. Lavine: J. Appl. Phys. 40, 409 (1969)

26. S.-C. Yu, I.L. Spain, E.F. Skelton: J. Appl. Phys. 49, 4741 (1978)

27. R.J. Nelmes, M.I. McMahon: Phys. Rev. Lett. 77, 663 (1996)

28. G.G. Gromov, V.V. Kapaev, Yu.V. Kopaev, K.V. Rudenko: Zh. Eksp. Teor. Fiz. 94, 101 (1988)

29. S.C. Howells, L.A. Schlie: Appl. Phys. Lett. 69, 550 (1996)

30. V. Chazapis, H.A. Blom, K.L. Vodopyanov, A.G. Norman, C.C. Phillips: Phys. Rev. B 52, 2516 (1995)

31. D.E. Aspnes, A.A. Studna: Phys. Rev. B 27, 985 (1983)

32. D.D. Lide (Ed.): CRC Handbook of Chemistry and Physics (CRC Press, Boca Raton 1993)

33. F. Vetelino, S.P. Gaur, S.S. Mitra: Phys. Rev. B 5, 2360 (1972)

34. J. Larsson, P.A. Heimann, Z. Chang, P.H. Bucksbaum, E. Judd, H.C. Kapteyn, R.W. Lee, X. Liu, A. Machacek, M.M. Murnane, H.A. Padmore, P.J. Schuck, B. Shan, J.S. Wark, R.W. Falcone: Opt. Lett. 22, 1012 (1997)

35. R. Yen, J.M. Liu, H. Kurz, N. Bloembergen: Appl. Phys. A 27, 153 (1982)

36. P.H. Bucksbaum, J. Bokor: Phys. Rev. Lett. 53, 182 (1984)

37. R. Keller, T. Renner, D.J. Massoletti: Proceedings of the 7th Beam Instrumentation Workshop, Argonne, IL, May 6-7, 1996

38. C.R. Wie, T.A. Tombrello, T. Vreeland Jr.: J. Appl. Phys. 59, 3743 (1986) 